\title{
Feasibility of adaptive computerized working memory and math intervention in 7-year-old children born preterm
}

\begin{abstract}
Katharina M. Heuser ${ }^{1}$, Britta Huening², Gisela Axmacher-Bopp¹, Claudia Roll3 ${ }^{3}$, Dariusz Michna ${ }^{4}$, Thomas Hoehn ${ }^{5}$, Norbert Teig ${ }^{6}$, Francisco Brevis ${ }^{7}$, Antonia Zapf ${ }^{8}$, Peter Bartmann ${ }^{9}$, Dieter Wolke ${ }^{10}$, Ursula Felderhoff-Mueser ${ }^{2}$, \& Julia Jaekel ${ }^{1,10,11}$

${ }^{1}$ Ruhr-Universität Bochum, Germany; ${ }^{2}$ Universitätsklinikum Essen, Germany; ${ }^{3}$ Vestische Kinder- und Jugendklinik Datteln, Germany; ${ }^{4}$ ElisabethKrankenhaus Essen, Germany; ${ }^{5}$ Universitätsklinikum Düsseldorf, Germany; ${ }^{6}$ Universitätsklinikum Bochum, Germany; ${ }^{7}$ Sana-Kliniken Duisburg, Germany; ${ }^{8}$ Universitätsklinikum Hamburg-Eppendorf, Germany; ${ }^{9}$ Universitätsklinikum Bonn, Germany; ${ }^{10}$ University of Warwick, UK; ${ }^{11}$ University of Tennessee Knoxville, USA
\end{abstract}

WHAT IS KNOWN: Computerized trainings may help decrease preterm children's risk for academic underachievement, but feasibility of such interventions at school age is not known.

OBJECTIVE: We aimed to evaluate preterm children's and their parents' motivations and the general feasibility of adaptive computerized working memory training (Cogmed(C) versus an openaccess math training (XtraMath@).

WHAT THIS STUDY ADDS: Our findings suggest that homebased delivery of both computerized trainings as intervention for school-aged children born preterm is feasible; however, XtraMath $\odot$ may be easier to implement into everyday life.

\section{RESULTS}

Overall, feasibility of both trainings was high. For example, children reported "the training was fun" (Mean (SD) Cogmed(C: 2.55 (0.69), XtraMath@: $2.58(0.72)$ ); parents rated integration into everyday life as easy (Cogmed(C: 2.79 (0.69), XtraMath@: 2.97 (0.61)).

Bootstrapped independent-samples $t$-tests showed that, on average, parents in the XtraMath $\odot$ group reported significantly higher general feasibility of the training compared with the Cogmed@ group (see Table 1), representing a medium-sized effect, $d=0.56$.

Table 1. Comparison of online training programs

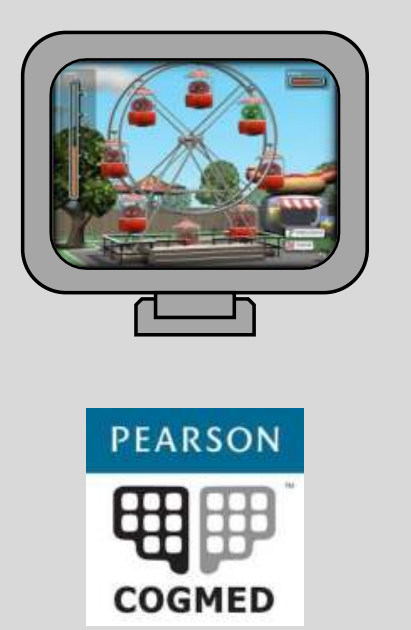

\begin{tabular}{|c|c|c|c|c|c|}
\hline & \multicolumn{2}{|c|}{ Online training program } & \multirow[b]{2}{*}{$\begin{array}{c}\text { Mean } \\
\text { difference }\end{array}$} & \multirow[b]{2}{*}{$\mathrm{BCa} 95 \% \mathrm{Cl}$} & \multirow[b]{2}{*}{$p$} \\
\hline & $\begin{array}{c}\text { Cogmed@ } \\
n=29\end{array}$ & $\begin{array}{c}\text { XtraMath } \odot \\
n=31\end{array}$ & & & \\
\hline Child's sex - male & $15(51.7 \%)$ & $15(48.4 \%)$ & & & .796 \\
\hline Gestational age, weeks & $33.00(1.95)$ & $32.68(2.17)$ & 0.32 & $(-0.67-1.28)$ & .547 \\
\hline Age, years & $7.07(0.45)$ & $7.17(0.31)$ & -0.10 & $(-0.29-0.09)$ & .316 \\
\hline Child report: Training Motivation & $-0.04(0.56)$ & $0.04(0.63)$ & -0.08 & $(-0.35-0.20)$ & .596 \\
\hline Parent report: Training Satisfaction & $-0.06(0.64)$ & $0.04(0.59)$ & -0.10 & $(-0.44-0.23)$ & .545 \\
\hline Parent report: Child's Motivation & $0.03(0.61)$ & $-0.03(0.73)$ & 0.06 & $(-0.24-0.36)$ & .694 \\
\hline Parent report: General Feasibility & $-0.26(0.91)$ & $0.23(0.83)$ & -0.49 & $(-0.90--0.08)$ & .037 \\
\hline
\end{tabular}

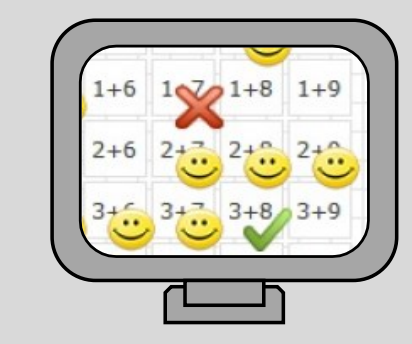

XtraMath

COGMED

\section{METHODS}

Figure 1. Randomization Flow-Chart

As part of a prospective, randomized-controlled, multi-center trial in Germany, preterm children $(28-35+6$ weeks of gestation; stratified by gestational age and sex) participated in one of two adaptive computerized trainings (Cogmed(C) versus XtraMath(C) at home for 5 days/week for 5 weeks during their first year of school (Figure 1).

At the post-training assessment, $n=60$ children answered questions adapted from the Intrinsic Motivation Inventory [1] (e.g., "I liked this training very much."). In addition, $n=59$ parents completed questions regarding training motivation, satisfaction, and general feasibility (e.g., "Did your child have fun performing the training?"). Child and parent responses were z-standardized and averaged into index scales (Cronbach's $\alpha \geq .74$ ).

Please have a look at our related e-poster: Preterm children's long-term academic performance after adaptive computerized training: A randomized controlled trial by Jaekel et al.



\section{CONCLUSION}

Our findings indicate that computerized trainings represent a motivating and feasible avenue towards intervention for school-aged preterm children. Compared with Cogmed(C), the adaptive, openaccess XtraMath( training may be easier to implement into everyday life, which may facilitate regular practice and fidelity. 